Review

\title{
Oncogenic virus-induced aerobic glycolysis and tumorigenesis
}

\author{
Li Yu ${ }^{1, *}, \otimes$, Xun Chen ${ }^{2,}{ }^{*}$, Liantang Wang ${ }^{1}$, Shangwu Chen ${ }^{3, \otimes}$ \\ 1. Department of Pathology, the First Affiliated Hospital, Sun Yat-sen University, Guangzhou 510080, People's Republic of China. \\ 2. Guanghua School and Hospital of Stomatology, Sun Yat-sen University, Guangzhou 510055, People's Republic of China. \\ 3. Guangdong Key Laboratory of Pharmaceutical Functional Genes, MOE Key Laboratory of Gene Function and Regulation, State Key Laboratory for \\ Biocontrol, Department of Biochemistry, School of Life Sciences, Sun Yat-sen University, Guangzhou 510275, People's Republic of China. \\ * The two authors contributed equally to this work.
}

$\triangle$ Corresponding authors: Li Yu, Department of Pathology, the First Affiliated Hospital, Sun Yat-sen University, Guangzhou 510080, People's Republic of China. Tel.: 86-20-87755766-8864, Fax: 86-20-87331780, E-mail: yuli5@mail.sysu.edu.cn; Shangwu Chen, Department of Biochemistry, School of Life Sciences, Sun Yat-sen University, Guangzhou 510275, People's Republic of China, Tel.: 86-20-39332958, Fax: 86-20-39332950, E-mail: lsschshw@mail.sysu.edu.cn.

(C) Ivyspring International Publisher. This is an open access article distributed under the terms of the Creative Commons Attribution (CC BY-NC) license (https://creativecommons.org/licenses/by-nc/4.0/). See http://ivyspring.com/terms for full terms and conditions.

Received: 2018.05.15; Accepted: 2018.08.15; Published: 2018.09.08

\begin{abstract}
Enhanced glycolysis under normoxic conditions is known as aerobic glycolysis or the Warburg effect and is a hallmark of many tumors. Viral infection may also induce aerobic glycolysis as it is required for replication and survival. Tumor viruses inducing aerobic glycolysis and lactate production during latent infection suggest a potential role of virus-induced glycolysis in tumorigenesis. Virus or virus-encoded proteins regulate glucose uptake and lactate export, increase the activity of glycolytic enzymes, and modulate glucose metabolic signals. Accumulating evidence suggests that virus-induced glycolysis may facilitate cell growth, transformation, migration, and invasion, but its significance in tumorigenesis remains unclear. We summarize the effects of oncogenic viruses on the metabolic shift to aerobic glycolysis and discuss the possible association of this metabolic reprogramming with tumor development and progression.
\end{abstract}

Key words: metabolic reprogramming, virus-induced glycolysis, aerobic glycolysis, tumorigenesis, oncogenic virus.

\section{Introduction}

In glycolysis, a molecule of glucose degrades into two pyruvate molecules. Tumor cells exhibit enhanced glycolysis even when the ambient oxygen supply is sufficient, a phenomenon known as aerobic glycolysis or the Warburg effect. Similar to the process in tumors, viral infections also induce aerobic glycolysis. Many intermediates of the glycolytic pathway are significantly increased following viral infection [1-3], dependent on viral protein expression [4]. Some viruses induce glycolysis for replication [3]. Viruses also use a metabolic shift to counter the reactive oxygen species (ROS) produced by the host in response to virus infection $[5,6]$. The mechanism of virus-induced glycolysis is believed to be virus species-specific [4]. Importantly, many viruses, including tumor viruses, induce aerobic glycolysis and lactate production during latent infections [7-9], suggesting a potential role of virus-induced glycolysis in tumorigenesis. We review information on oncogenic virus induction of aerobic glycolysis and the possible association of this metabolic reprogramming with tumor development and progression.

\section{Involvement of oncogenic viruses in regulation of the Warburg effect}

Similar to cancer cells, virus-infected cells exhibit increased glycolysis [8, 10, 11]. In addition to infectious diseases, many viruses contribute to the process of cancerogenesis and are known as tumor viruses. Both DNA and RNA viruses exhibit oncogenic potential. Generally, DNA tumor viruses 
include adenoviruses, herpes viruses, papilloma viruses, and polyomavirus [12]. Accumulating evidence suggests that oncogenic viral proteins contribute to aerobic glycolysis during tumorigenesis, but the underlying mechanisms remain unclear [13]. Some viral genes that facilitate tumor development have been identified [12]. Many viruses have been shown to activate and re-program cell metabolism [14]. Oncogenic viruses contribute to the metabolic effects of cell transformation. Viral oncogenes interact with and regulate a variety of cell signaling pathways and induce metabolic alterations during cell transformation (Table 1) [15]. Current data suggest that viruses either initiate metabolic events contributing to transformation or activate metabolic pathways responsible for tumor progression. Oncogenic viruses or virus-encoded proteins alter host cell metabolism by modifying the expression and activity of glucose transporters and glycolytic enzymes and modulating the function of key signaling molecules involved in glucose metabolism (Fig. 1). Many viruses, including Epstein-Barr virus (EBV), human cytomegalovirus (HCMV), hepatitis B virus (HBV), hepatitis $C$ virus $(\mathrm{HCV})$, herpes simplex virus (HSV), human papilloma virus (HPV), and Kaposi's sarcoma-associated herpesvirus (KSHV) have been shown to reprogram cell metabolism, supporting replication as well as facilitating cancer development and progression.

Table 1. Oncogenic viruses and their regulation of aerobic glycolysis.

\begin{tabular}{|c|c|c|c|c|c|}
\hline \multirow[t]{2}{*}{ Viruses } & \multirow{2}{*}{$\begin{array}{l}\text { Oncogenic } \\
\text { genes/proteins }\end{array}$} & \multicolumn{3}{|c|}{ Effector factors } & \multirow[t]{2}{*}{ References } \\
\hline & & Transporters & $\begin{array}{l}\text { Glycolytic } \\
\text { enzymes }\end{array}$ & Signal molecules & \\
\hline AV & E4ORF1 & & $\begin{array}{l}\text { GAPDH, } \\
\text { HK2, } \\
\text { LDHA, } \\
\text { PFK1 }\end{array}$ & Myc & [45] \\
\hline \multirow[t]{8}{*}{ EBV } & LMP1 & GLUT1 & & mTORC1/NF-kB & {$[13]$} \\
\hline & LMP1 & & HK2 & c-Myc & [9] \\
\hline & LMP1 & & $\begin{array}{l}\text { LDHA, } \\
\text { PKM2 }\end{array}$ & FGFR1, HIF-1a & [65] \\
\hline & LMP1 & GLUT1 & HK2 & HoxC8 & [66] \\
\hline & $\begin{array}{l}\text { EBNA3, } \\
\text { EBNA5 }\end{array}$ & & & $\begin{array}{l}\text { PHD1, PHD2, } \\
\text { HIF-1a }\end{array}$ & [55] \\
\hline & & GLUT1 & & & [26] \\
\hline & & GLUT1 & & IKK $\beta /$ Akt & [27] \\
\hline & & MCT4 & & & [26] \\
\hline FSV & & GLUT1 & & & [16] \\
\hline \multirow[t]{3}{*}{ HBV } & Pre-S2 & GLUT1 & & mTOR/YY1/Myc & [24] \\
\hline & $\mathrm{HBx}$ & & G6PD & Nrf2 & [67] \\
\hline & $\mathrm{HBc}$ & & $\begin{array}{l}\text { ALDOC, } \\
\text { PCK1 }\end{array}$ & MLX & {$[68]$} \\
\hline \multirow[t]{2}{*}{ HCMV } & & GLUT4 & & & {$[22]$} \\
\hline & & & PFK1 & & {$[2,4]$} \\
\hline \multirow[t]{5}{*}{$\mathrm{HCV}$} & NS5B & & PKM2 & & {$[41]$} \\
\hline & NS5A & & HK2 & & [43] \\
\hline & & & PDK & & [50] \\
\hline & & & $\begin{array}{l}\text { LDHA, } \\
\text { HK1 }\end{array}$ & HIF-1a & [54] \\
\hline & & & & $\mathrm{HNF} 4 \mathrm{a}$ & [69] \\
\hline HIV1 & & GLUT1, & & & {$[17]$} \\
\hline
\end{tabular}

\begin{tabular}{|c|c|c|c|c|c|}
\hline \multirow[t]{2}{*}{ Viruses } & \multirow{2}{*}{$\begin{array}{l}\text { Oncogenic } \\
\text { genes/proteins }\end{array}$} & \multicolumn{3}{|c|}{ Effector factors } & \multirow[t]{2}{*}{ References } \\
\hline & & Transporters & $\begin{array}{l}\text { Glycolytic } \\
\text { enzymes }\end{array}$ & Signal molecules & \\
\hline & & GLUT3 & & & \\
\hline \multirow[t]{8}{*}{ HPV } & E2 & & & ROS, HIF-1a & [53] \\
\hline & E6 & & & VHL, HIF-1a & [52] \\
\hline & E6 & & & miR-34a & [63] \\
\hline & E6 & SGLT1 & & & [20] \\
\hline & E7 & & PKM2 & & [36] \\
\hline & E6/E7 & & HK2 & c-Myc, LKB1 & [44] \\
\hline & E6/E7 & GLUT1 & & HIF-1a & [21] \\
\hline & & $\begin{array}{l}\text { MCT4, } \\
\text { CD147 }\end{array}$ & & & [33] \\
\hline HSV1 & & & PFK1 & & [47] \\
\hline $\mathrm{JCV}$ & T-antigen & & $\begin{array}{l}\text { HK2, } \\
\text { TALDO1 }\end{array}$ & & [46] \\
\hline \multirow[t]{9}{*}{ KSHV } & $\begin{array}{l}\text { miRNAs, } \\
\text { vFLIP }\end{array}$ & $\begin{array}{l}\text { GLUT1, } \\
\text { GLUT3 }\end{array}$ & & NF-kB & [28] \\
\hline & miRNAs & & & $\begin{array}{l}\text { PDH1, HSPA9, } \\
\text { HIF-1a }\end{array}$ & [64] \\
\hline & LANA & CD147 & & & [32] \\
\hline & K5 & & & Akt, Erk1/2 & [71] \\
\hline & & GLUT1 & & & [25] \\
\hline & & & PKM2 & HIF-1a & [40] \\
\hline & & & & HIF-1a & [57] \\
\hline & & GLUT3 & HK2 & & [7] \\
\hline & & & & PI3K/Akt & [70] \\
\hline RSV & Src & & PKM2 & & [38] \\
\hline VACV & $\mathrm{C} 16$ & GLUT1 & PDK1 & PHD2, HIF-1a & [56] \\
\hline
\end{tabular}

ALDOC, aldolase $\mathrm{C} ; \mathrm{AV}$, adenovirus; $\mathrm{EBV}$, Epstein-Barr virus; FGFR1, fibroblast growth factor receptor 1; FSV, Fujinama sarcoma virus; GLUT, glucose transporter; $\mathrm{HBc}, \mathrm{HBV}$ core protein; $\mathrm{HBV}$, hepatitis B virus; $\mathrm{HBx}, \mathrm{HBV} X$ protein; $\mathrm{HCMV}$, human cytomegalovirus; $\mathrm{HCV}$, hepatitis $\mathrm{C}$ virus; $\mathrm{HIF-1a}$, hypoxia inducible factor-1a; HK2, hexokinase 2; HPV, human papilloma virus; HSV, herpes simplex virus; JCV, John Cunningham virus; KSHV, Kaposi's sarcoma-associated herpesvirus; LANA, latency-associated nuclear antigen; LDH, lactate dehydrogenase; LKB1, liver kinase B1; LMP1, EBV-encoded latent membrane protein 1; MCT, monocarboxylate transporter; MLX, Max-like protein X; PCK1, phosphoenolpyruvate carboxykinase; PDK, pyruvate dehydrogenase kinase; PFK1, 6-phosphofructo-1-kinase; PHDs, prolylhydroxylase; PKM2, pyruvate kinase M2; ROS, reactive oxygen species; VACV, vaccinia virus; VHL, von Hippel-Lindau tumor suppressor; RSV, Rous sarcoma virus; SGLT1, Na+-glucose cotransporter; TALDO1, transaldolase-1.

\section{Regulation of glucose uptake and lactate export by tumor cells}

Transport of glucose into the cell is the basis of its metabolism, which controls the glycolytic flux in virus-infected cells. With glucose channeled to the glycolysis pathway, uptake of extracellular glucose must be enhanced to meet the needs of the increased metabolic rate. The expression of glucose transporters, as well as their trafficking to the plasma membrane, influences cell glucose uptake. In early studies, it was demonstrated that infection of cells by Fujinama sarcoma virus (FSV), HIV1, SV40, and polyomavirus promoted expression of glucose transporter 1(GLUT1) and GLUT3 or their membrane trafficking [16-19]. Human papilloma virus E6 expression significantly increases the protein abundance of $\mathrm{Na}^{+}$-glucose cotransporter (SGLT1) in the cell membrane [20]. Overexpression of HPV E6 and E7 in lung cancer cells significantly promotes hypoxia inducible factor-1a (HIF-1a)-mediated expression of GLUT1 [21]. Human cytomegalovirus induces GLUT4 expression, glucose uptake [22], and consequent glycolysis via activation of 6-phosphofructo-1-kinase (PFK1) [2, 4]. Hepatitis B 


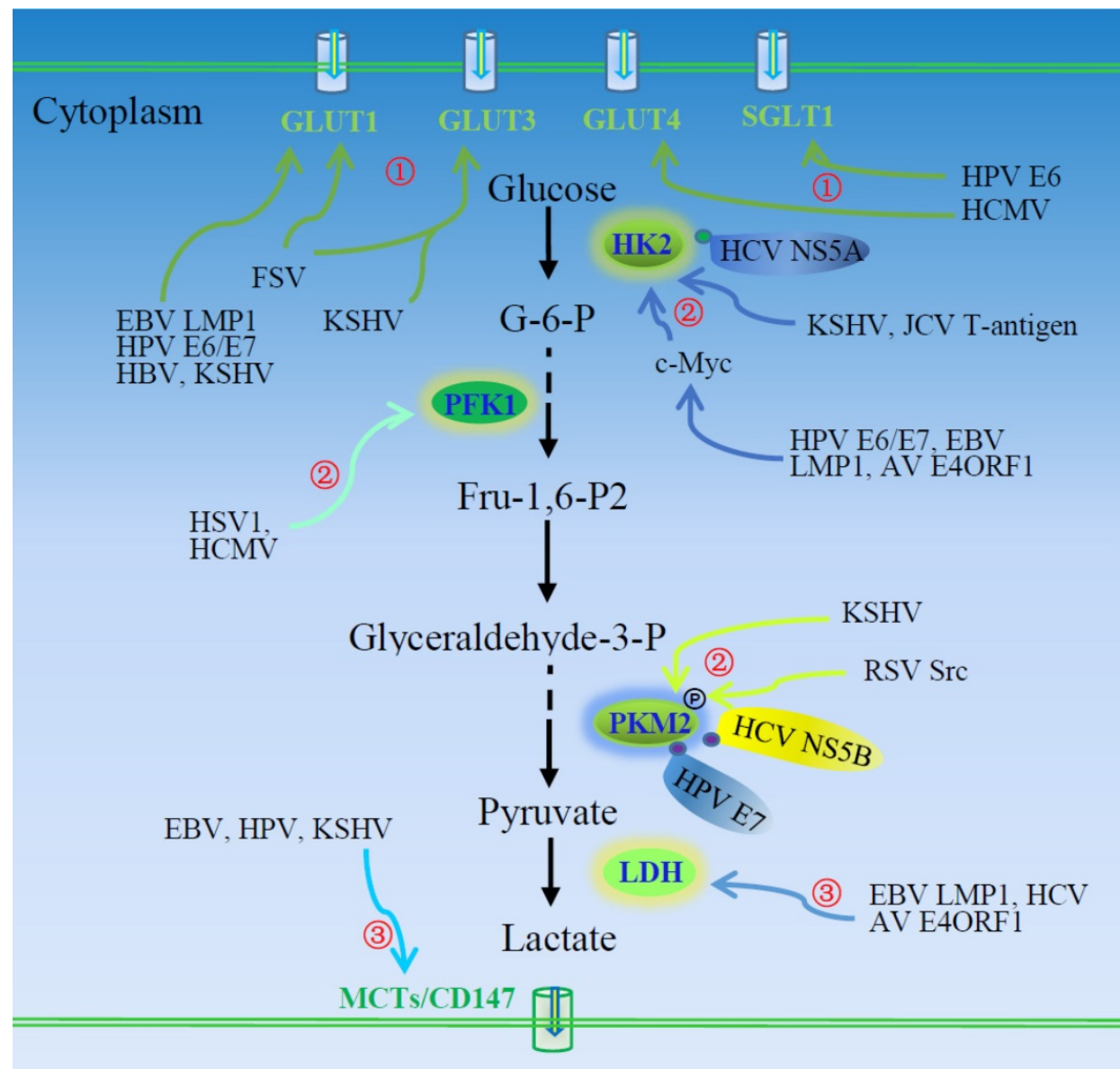

Figure 1. Oncogenic viruses induce aerobic glycolysis. (1)Viruses or virus-encoded proteins enhance glucose uptake and consumption via inducing expression of glucose transporters or their plasma membrane trafficking. (2) Oncogenic viruses also regulate the expression and activity of three rate-limiting enzymes of the glycolytic pathway, $\mathrm{HK2}$ PFK1, and PKM2. (3)Virus-induced LDH activation and MCT expression facilitate the production and secretion of lactate. AV, adenovirus; EBV, Epstein-Barr virus; FSV, Fujinama sarcoma virus; GLUTs, glucose transporters; HBV, hepatitis B virus; HCMV, human cytomegalovirus; HCV, hepatitis C virus; HK2, hexokinase 2; HPV, human papilloma viruses; HSV, herpes simplex virus; JCV, John Cunningham virus; KSHV, Kaposi's sarcoma-associated herpesvirus; LDH, lactate dehydrogenase; LMPI, EBV-encoded latent membrane protein 1; MCT, monocarboxylate transporter; PFK1, 6-phosphofructo-1-kinase; PKM2, pyruvate kinase M2; RSV, Rous sarcoma virus.

virus pre-S/S gene mutation increases oncogenic potential [23], and HBV pre-S2 mutant-induced mTOR activation facilitates the metabolic switch in HBV tumorigenesis. The pre-S2 mutant activates the mTOR/YY1/Myc/GLUT1 signaling cascade. Activation of mTOR signal cascade promotes GLUT1 translocation to the plasma membrane and aerobic glycolysis [24]. Glucose transporter 1 membrane trafficking is also increased in KSHV-infected THP-1 cells [25]. Functional changes in the glucose transport system may be critical to virus-induced oncogenesis.

Epstein-Barr virus causes a number of malignancies including Burkitt's lymphoma and nasopharyngeal carcinoma (NPC). It induces GLUT1 expression [26] and its translocation [12, 27]. Epstein-Barr virus encoded latent membrane protein 1 (LMP1) exhibits tumorigenic transformation properties. Latent membrane protein 1 activates mTORC1 and subsequent downstream NF-kB signaling, inducing GLUT1 transcription and promoting aerobic glycolysis [13]. Loss of GLUT1 impairs the tumorigenic growth property of
LMP1-expressing nasopharyngeal epithelial cells. Latent membrane protein 1 promotes aerobic glycolysis and tumorigenic growth of NPC cells via the mTORC1/NF-kB/GLUT1 pathway, which may provide a target for the therapy of EBV-induced NPC [13].

Kaposi's sarcoma-associated herpesvirus (KSHV) is the etiologic agent of Kaposi's sarcoma (KS), the most common tumor of AIDS patients world-wide. Kaposi's sarcoma-associated herpesvirus-induced metabolic changes are similar to those in tumors [7]. Its latent infection of endothelial cells induces GLUT3 expression and glucose uptake and upregulates glycolytic enzyme hexokinase 2 (HK2), leading to increased lactic acid production. Thus, KSHV infection induces aerobic glycolysis. Inhibition of glycolysis induces apoptosis of KSHVinfected endothelial cells [7]. Aerobic glycolysis is usually essential to rapid growth of tumors; however, under glucose deprivation, KSHV inhibits glycolysis and oxidative phosphorylation, as opposed to inducing glycolysis [28]. This is suggested to act in 
stress and promote cell survival and transformation [28].

Lactate can regulate dendritic cell activation and antigen presentation, facilitating tumor cell evasion of the immune system $[29,30]$. Thus, lactate produced via aerobic glycolysis may be critical to tumor metastasis. Monocarboxylate transporters (MCT) transport lactate into the extracellular environment. Cluster of differentiation 147 (CD147) is required for plasma membrane expression and function of MCT1 and MCT4 [31]. Kaposi's sarcoma-associated herpesvirus or viral latency-associated nuclear antigen (LANA) upregulates the expression of CD147 and induces invasion of primary endothelial cells [32]. Human papilloma virus transfection leads to upregulation of MCT4 and CD147 [33]. Monocarboxylate transporter 4 expression is up-regulated in Burkitt's lymphoma cell lines [26]. It is necessary to investigate the role of enhanced production and export of lactate in virus-associated tumor progression.

\section{Promotion of glycolytic enzyme activity}

Many glycolytic enzymes are up-regulated in viral-infected cells, as shown by proteomic profiling [34]. Pyruvate kinase M2 (PKM2) is a predominant isoform of pyruvate kinase in proliferating tumor cells, present as a highly active tetramer and as a dimer with low affinity for phosphoenolpyruvate. The alteration in isoform can regulate the glycolysis. As a rate-limiting enzyme in glycolysis, PKM2 is highly regulated by many viruses: Human papilloma virus E7 can bind to PKM2 in the cytoplasm, inducing structural change from the more active tetramer to the less active dimer $[35,36]$. Although inhibiting effects are reported in a few studies, the majority of viruses promote glycolytic enzyme activity. The oncogenic protein Src of Rous sarcoma virus (RSV) can phosphorylate PKM2 and increase its activity [37, 38]. Hexokinase and phosphofructokinase are also phosphorylated in cells infected by RSV [39]. Hypoxia inducible factor-1a promotes the Warburg effect in KSHV-infected endothelial cells by upregulating PKM2 [40]. Lactate production is increased in the KSHV-infected cell in a PKM2-dependent manner. Pyruvate kinase M2 promotes the tumorigenic potential of the KSHV oncogene and the angiogenic potential of infected cells. Hepatitis C virus NS5B, the HCV RNA-dependent RNA polymerase, interacts with PKM2, and PKM2 downregulation inhibits HCV replication, implying a role of PKM2 in metabolism-dependent viral replication [41].

Hexokinase 2 is another critical glycolytic enzyme impacted by oncogenic viruses. Hepatitis viruses modulate glycolysis, showing tumorigenic potential. Woodchuck hepatitis virus-infected cells exhibit enhanced hexokinase activity [42]. Glucose consumption and lactate production is increased in $\mathrm{HCV}$-infected cells. Hepatitis C virus NS5A can interact directly with cellular HK2, the first rate-limiting enzyme of glycolysis, activating HK2 and enhancing glucose consumption and lactate secretion, which may contribute to an increased glycolysis rate during $\mathrm{HCV}$ infection [43]. Human papilloma virus16 E6/E7 facilitates the expression of HK2 through elevated c-Myc [44]. Expression of liver kinase B1 (LKB1) reduces HK2 and glycolysis. It is believed that LKB1 retards HPV-stimulated aerobic glycolysis and tumor progression. Liver kinase B1 is inactivated through mutation in many cancer types, including HPV-related cervical cancer [44]. The c-Myc induced-up-regulation of HK2 is required for EBV LMP1-mediated reprogramming of glucose metabolism in NPC cells [9], and the up-regulation of HK2 facilitates aerobic glycolysis and cell proliferation. Latent membrane protein 1 is the key regulator of EBV-mediated glycolysis in NPC cells. The exogenous overexpression of EBV LMP1 significantly increases glycolysis. Targeting EBV LMP1-mediated glycolysis sensitizes nasopharyngeal carcinoma to radiation therapy [9]. The adenovirusencoded E4ORF1 binds to c-Myc and induces expression of glycolytic enzymes such as HK2, PFK1, glyceraldehyde-3-phosphate dehydrogenase (GAP $\mathrm{DH})$, and lactate dehydrogenase A (LDHA), promoting glycolysis [45]. The human polyomavirus John Cunningham virus (JCV) T-antigen regulates expression of the glycolytic enzymes HK2 and transaldolase-1 (TALDO1) in medulloblastoma cells [46].

Herpes simplex virus type 1 infection increases glucose uptake and lactate production via PFK1 synthesis and activation [47]. Phosphofructokinase-2 (PFK2) activity and level of fructose 2,6-bisphosphate have been reported to be increased in RSV-infected chick embryo fibroblasts [48, 49]. Hepatitis C virus replication requires host-produced macromolecules, which promotes host cell metabolic reprogramming to aerobic glycolysis mediated in part by increased activity of pyruvate dehydrogenase kinase (PDK), a mitochondrial enzyme that promotes aerobic glycolysis [50].

\section{Modulation of glucose metabolic signaling}

Several viruses clearly elicit the Warburg effect during viral infection. Some viral oncoproteins modulate the glycolytic pathway in infected cells through interaction with cellular signaling molecules. 


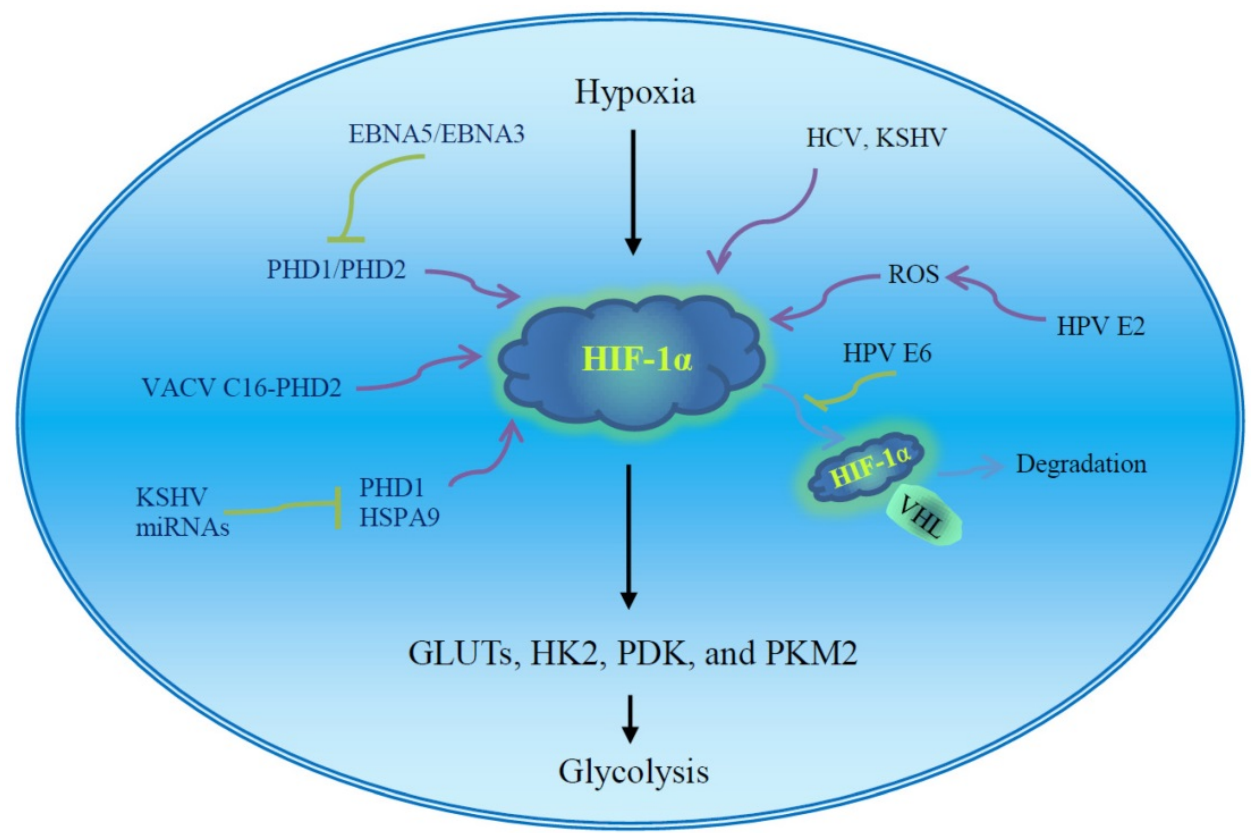

Figure 2. The regulation of virus and virus-encoded proteins on the HIF-1 $\alpha$. The stability and activity of HIF-1 $\alpha$ are regulated by post-translational modifications. HIF-1 $\alpha$ is rapidly degraded under normoxia via the VHL-mediated ubiquitin-proteasome pathway. PHD-mediated hydroxylation promotes the association of VHL and HIF-1a. The ROS inhibits PHDs and stabilizes the HIF-1 $\alpha$. The virus and virus-encoded proteins facilitate HIF-1 $\alpha$ accumulation under normoxia via disrupting the association of HIF-1 $\alpha$ and VHL or PHDs, inducing ROS production, and possible unknown mechanisms. The enhanced HIF-1a potentiates the transcription of GLUTs and glycolytic enzymes and induces aerobic glycolysis. Regulation of viral oncoproteins on HIF-1a transcriptional activity has been well described in Figure 2 of the review paper [51]. EBV, Epstein-Barr virus; GLUTs, glucose transporters; HCV, hepatitis C virus; HIF-1a, hypoxia inducible factor-1a; HK2, hexokinase 2; HPV, human papilloma virus; HSP, heat shock protein; KSHV, Kaposi's sarcoma-associated herpesvirus; PDK, pyruvate dehydrogenase kinase; PHD, prolylhydroxylase; PKM2, pyruvate kinase M2; ROS, reactive oxygen species; VACV, vaccinia virus; VHL, von Hippel-Lindau tumor suppressor.

Hypoxia inducible factor-1a is a master regulator of aerobic glycolysis and its role in viral carcinogenesis has been well reviewed [51]. The expression and stability of HIF-1a are influenced by multiple viral proteins (Fig. 2). Human papilloma virus E6 increases cellular HIF-1a level [52]. Human papilloma virus 16 E6 promotes hypoxia-induced Warburg effect through disrupting the association of HIF-1a and von Hippel-Lindau tumor suppressor (VHL), an E3 ligase. The dissociation of HIF-1a from VHL attenuates VHL-mediated HIF-1a ubiquitination and leads to HIF-1a accumulation [52]. Cytoplasmic oncogenic HPV18 E2 localizes to mitochondrial membranes and increases production of mitochondrial ROS, stabilizing HIF-1 $\alpha$ and increasing glycolysis [53]. Thus, high-risk HPV E2-mediated Warburg effect or the modification of cell metabolism could play a role in carcinogenesis [53]. Hepatitis C virus infection inhibits oxidative phosphorylation and promotes glycolysis [54], and HCV proteins induce HIF-1a stabilization under normoxic conditions, which facilitates the expression of glycolytic enzymes such as LDHA and HK1. Hepatitis C virus-associated mitochondrial dysfunction promotes HIF-1amediated glycolytic adaptation. This knowledge provides new insight into the pathogenesis of chronic hepatitis C and, possibly, HCV-related hepatocellular carcinoma [54]. The aerobic glycolytic pathway is activated in EBV-infected lymphoblastoid cells.
Epstein-Barr virus-encoded nuclear antigen 5 (EBNA5) binds to and inactivates prolylhydroxylase 1 (PHD1) and EBNA3 binds to PHD2, consequently inhibiting hydroxylation and ubiquitination-mediated degradation of HIF-1a and leading to transactivation of aerobic glycolytic genes and production of high levels of lactate and pyruvate [55]. Vaccinia virus (VACV) infection stabilizes HIF-1a and promotes HIF-1a translocation to the nucleus, activating HIF-responsive genes such as vascular endothelial growth factor (VEGF), GLUT1, and PDK1. Vaccinia virus protein $\mathrm{C} 16$ directly binds the human oxygen sensing enzyme PHD2 and inhibits PHD2-dependent hydroxylation of HIF-1a [56]. HIF-1a is also significantly increased in the KSHV latent-infected endothelial cells [57].

The p53 tumor suppressor is a critical regulator of glycolysis that down-regulates the expression of glucose transporters and decreases glycolytic metabolism [58, 59]. It is regulated by AMPK in response to metabolic stress. Many viral oncoproteins, including mouse polyomavirus large T-antigen, HPV E6, and EBV nuclear antigen (EBNA) 3C [60-62], regulate the p53 pathway, suggesting that virus oncoprotein-mediated p53 inactivation may enhance glucose uptake and accelerate glycolysis in cancer cells.

Virus miRNAs may serve as modulators of glycolysis in infected cells and contribute to tumor 
progression. miR-34a inhibits glucose consumption and lactate production in cervical cancer cells [63]. Human papilloma virus E6 reduces miR-34a expression, promoting the Warburg effect and consequent progression of cervical cancer [63]. The expression of KSHV miRNA decreases oxygen consumption and increases glucose uptake and lactate secretion, inducing aerobic glycolysis in infected cells [64]. The KSHV miRNAs induce the metabolic switch through targeting PHD1 and mitochondrial heat shock protein HSPA9, leading to stabilization of HIF-1a and reduction of mitochondria biogenesis. The metabolic shift provides a growth advantage in low oxygen conditions, which is critical to the latent viral infection as well as the survival of tumor cells [64].

Epstein-Barr virus LMP1 plays an important role in the pathogenesis of nasopharyngeal carcinoma (NPC). Latent membrane protein 1 induces expression and activation of fibroblast growth factor receptor 1 (FGFR1) [65] and repression of several Hox genes [66]. Inhibition of FGFR1 signaling attenuates LMP1mediated aerobic glycolysis and malignant transformation of human nasopharyngeal epithelial cells [65]. Ectopic expression of HoxC8 down-regulates the expression of HK2 and GLUT1 and represses tumor progression in NPC cells [66]. The data indicate that LMP1-mediated metabolic reprogramming contributes to the development and progression of NPC.

Chronic HBV infection is associated with hepatocellular carcinoma (HCC). Hepatitis B virus $X$ protein $(\mathrm{HBx})$ stimulates the expression of glucose-6-phosphate dehydrogenase (G6PD) via activation of the transcription factor Nrf2, implying a potential effect of $\mathrm{HBV}$ on the reprogramming of glucose metabolism, which may be of importance in the development of HBV-associated hepatocarcinoma [67]. Glucose-6-phosphate dehydrogenase is the first and rate-limiting enzyme in the pentose phosphate pathway. It is highly expressed in chronic $\mathrm{HBV}$-infected human liver and HBV-associated liver cancer. Proteomics and metabolomics studies indicate that $\mathrm{HBV}$ core protein $(\mathrm{HBC})$ significantly induces glycolysis in HCC cells. It may recruit Max-like protein $X(M L X)$, a transcription factor, and facilitate its translocation to the nucleus, where it promotes the expression of glycolytic enzymes, such as aldolase $C$ (ALDOC) and phosphoenolpyruvate carboxykinase (PCK1) [68].

In addition, HCV induces glycolysis by activation of HNF4a, a nuclear transcription factor [69]. Infection by KSHV is associated with primary effusion lymphoma (PEL), a unique subset of human B-cell non-Hodgkin lymphoma (B-NHL). Aerobic glycolysis is up-regulated in PEL in a PI3K/Akt-dependent manner [70]. Kaposi's sarcoma-associated herpesvirus K5, a viral E3 ubiquitin ligase, increases aerobic glycolysis and lactate production through alterations in growth factor signaling cascades [71].

\section{Conclusions}

It has long been believed that many viruses can induce malignant transformation of cells. The effects of oncogenic viruses on growth signaling pathways have been well studied, but the regulation of viruses and their encoded proteins on cellular metabolism has only begun to attract attention. Glucose metabolism is a main metabolic pathway of cell energy metabolism. Aerobic glycolysis is a hallmark of tumors. Although virus infection may lead to glycolysis, the significance of virus-induced glycolysis in tumorigenesis remains unclear. Obviously, virus-induced glycolysis is more than just a direct cellular response to infection [14]. Many oncogenic viruses induce metabolic alterations similar in most cancer cells, suggesting a role of virus-induced metabolism in cellular oncogenesis [14]. Currently available data demonstrate that viruses potentiate tumor formation and progression through driving expression of oncogenes or reprogramming glucose metabolism via regulation of glucose uptake, glycolytic flux, and energy production. Aerobic glycolysis provides an advantage to virus-associated tumor cells by facilitating growth even under conditions of nutrient and oxygen scarcity. However, the mechanism and significance of virus-mediated glycolysis remain to be elucidated. Currently available data have been mainly generated by studies of transformed cell lines, and interpretation is a challenge. The development of relevant animal models will be valuable in future studies. Understanding virus-induced metabolism reprogramming will aid in developing glycolysis-based therapeutic strategies for oncogenic virus-induced cancers.

\section{Acknowledgments}

This study was funded by the National Natural Science Foundation of China (No. 31670788 and No. 81172485), the Ph.D. Program Foundation of Ministry of Education of China (No. 20130171110007), and Open Fund of Guangdong Key Laboratory of Pharmaceutical Functional Genes (No.2014B030301 028 and No.2017B030314021).

\section{Competing Interests}

The authors have declared that no competing interest exists. 


\section{References}

1. Munger J, Bennett BD, Parikh A, Feng XJ, McArdle J, Rabitz HA, et al. Systems-level metabolic flux profiling identifies fatty acid synthesis as a target for antiviral therapy. Nat Biotechnol. 2008; 26: 1179-86.

2. Munger J, Bajad SU, Coller HA, Shenk T, Rabinowitz JD. Dynamics of the cellular metabolome during human cytomegalovirus infection. PLoS Pathog. 2006; 2: e132.

3. Fontaine KA, Sanchez EL, Camarda R, Lagunoff M. Dengue virus induces and requires glycolysis for optimal replication. J Virol. 2015; 89: 2358-66.

4. McArdle J, Schafer XL, Munger J. Inhibition of calmodulin-dependent kinase kinase blocks human cytomegalovirus-induced glycolytic activation and severely attenuates production of viral progeny. J Virol. 2011; 85: 705-14.

5. Chen IT, Lee DY, Huang YT, Kou GH, Wang HC, Chang GD, et al. Six hours after infection, the metabolic changes induced by WSSV neutralize the host's oxidative stress defenses. Sci Rep. 2016; 6: 27732.

6. Su MA, Huang YT, Chen IT, Lee DY, Hsieh YC, Li CY, et al. An invertebrate Warburg effect: a shrimp virus achieves successful replication by altering the host metabolome via the PI3K-Akt-mTOR pathway. PLoS Pathog. 2014; 10: e1004196.

7. Delgado T, Carroll PA, Punjabi AS, Margineantu D, Hockenbery DM, Lagunoff M. Induction of the Warburg effect by Kaposi's sarcoma herpesvirus is required for the maintenance of latently infected endothelial cells. Proc Natl Acad Sci U S A. 2010; 107: 10696-701.

8. Delgado T, Sanchez EL, Camarda R, Lagunoff M. Global metabolic profiling of infection by an oncogenic virus: KSHV induces and requires lipogenesis for survival of latent infection. PLoS Pathog. 2012; 8: e1002866.

9. Xiao L, Hu ZY, Dong X, Tan Z, Li W, Tang M, et al. Targeting Epstein-Barr virus oncoprotein LMP1-mediated glycolysis sensitizes nasopharyngeal carcinoma to radiation therapy. Oncogene. 2014; 33: 4568-78.

10. Corporeau C, Tamayo D, Pernet F, Quere C, Madec S. Proteomic signatures of the oyster metabolic response to herpesvirus OsHV-1 muVar infection. J Proteomics. 2014; 109: 176-87.

11. Chen IT, Aoki T, Huang YT, Hirono I, Chen TC, Huang JY, et al. White spot syndrome virus induces metabolic changes resembling the warburg effect in shrimp hemocytes in the early stage of infection. J Virol. 2011; 85: 12919-28.

12. Mushtaq M, Darekar S, Kashuba E. DNA tumor viruses and cell metabolism. Oxid Med Cell Longev. 2016; 2016: 6468342.

13. Zhang J, Jia L, Lin W, Yip YL, Lo KW, Lau VM, et al. Epstein-Barr virus-encoded latent membrane protein 1 upregulates glucose transporter 1 transcription via the mTORC1/NF-kappaB signaling pathways. J Virol. 2017; 91.

14. Sanchez EL, Lagunoff M. Viral activation of cellular metabolism. Virology. 2015; 479-480: 609-18.

15. Noch E, Khalili K. Oncogenic viruses and tumor glucose metabolism: like kids in a candy store. Mol Cancer Ther. 2012; 11: 14-23.

16. Birnbaum MJ, Haspel HC, Rosen OM. Transformation of rat fibroblasts by FSV rapidly increases glucose transporter gene transcription. Science. 1987; 235: 1495-8.

17. Sorbara LR, Maldarelli F, Chamoun G, Schilling B, Chokekijcahi S, Staudt L, et al. Human immunodeficiency virus type 1 infection of $\mathrm{H} 9$ cells induces increased glucose transporter expression. J Virol. 1996; 70: 7275-9.

18. Kitagawa K, Nishino H, Iwashima A. Analysis of hexose transport in untransformed and sarcoma virus-transformed mouse 3T3 cells by photoaffinity binding of cytochalasin B. Biochim Biophys Acta. 1985; 821: 63-6.

19. Young AT, Dahl J, Hausdorff SF, Bauer PH, Birnbaum MJ, Benjamin TL. Phosphatidylinositol 3-kinase binding to polyoma virus middle tumor antigen mediates elevation of glucose transport by increasing translocation of the GLUT1 transporter. Proc Natl Acad Sci U S A. 1995; 92: 11613-7.

20. Leiprecht N, Munoz C, Alesutan I, Siraskar G, Sopjani M, Foller M, et al. Regulation of $\mathrm{Na}(+)$-coupled glucose carrier SGLT1 by human papillomavirus 18 E6 protein. Biochem Biophys Res Commun. 2011; 404: 695-700.

21. Fan R, Hou WJ, Zhao YJ, Liu SL, Qiu XS, Wang EH, et al. Overexpression of HPV16 E6/E7 mediated HIF-1alpha upregulation of GLUT1 expression in lung cancer cells. Tumour Biol. 2016; 37: 4655-63.

22. Yu Y, Maguire TG, Alwine JC. Human cytomegalovirus activates glucose transporter 4 expression to increase glucose uptake during infection. J Virol. 2011; 85: 1573-80.

23. Lai MW, Liang KH, Lin WR, Huang $\mathrm{YH}$, Huang SF, Chen TC, et al. Hepatocarcinogenesis in transgenic mice carrying hepatitis $\mathrm{B}$ virus pre-S/S gene with the sW172* mutation. Oncogenesis. 2016; 5: e273.

24. Teng CF, Hsieh WC, Wu HC, Lin YJ, Tsai HW, Huang W, et al. Hepatitis B virus pre-S2 mutant induces aerobic glycolysis through mammalian target of rapamycin signal cascade. PLoS One. 2015; 10: e0122373.

25. Gonnella R, Santarelli R, Farina A, Granato M, D'Orazi G, Faggioni A, et al. Kaposi sarcoma associated herpesvirus (KSHV) induces AKT hyperphosphorylation, bortezomib-resistance and GLUT-1 plasma membrane exposure in THP-1 monocytic cell line. J Exp Clin Cancer Res. 2013; 32: 79.

26. Mushtaq M, Darekar S, Klein G, Kashuba E. Different mechanisms of regulation of the Warburg effect in lymphoblastoid and Burkitt lymphoma cells. PLoS One. 2015; 10: e0136142.

27. Sommermann TG, O'Neill K, Plas DR, Cahir-McFarland E. IKKbeta and NF-kappaB transcription govern lymphoma cell survival through AKT-induced plasma membrane trafficking of GLUT1. Cancer Res. 2011; 71: 7291-300.
28. Zhu Y, Ramos da Silva S, He M, Liang Q, Lu C, Feng P, et al. An oncogenic virus promotes cell survival and cellular transformation by suppressing glycolysis. PLoS Pathog. 2016; 12: e1005648.

29. Nasi A, Fekete T, Krishnamurthy A, Snowden S, Rajnavolgyi E, Catrina AI, et al. Dendritic cell reprogramming by endogenously produced lactic acid. J Immunol. 2013; 191: 3090-9.

30. Gottfried E, Kunz-Schughart LA, Ebner S, Mueller-Klieser W, Hoves S, Andreesen $\mathrm{R}$, et al. Tumor-derived lactic acid modulates dendritic cell activation and antigen expression. Blood. 2006; 107: 2013-21.

31. Halestrap AP, Meredith D. The SLC16 gene family-from monocarboxylate transporters (MCTs) to aromatic amino acid transporters and beyond. Pflugers Arch. 2004; 447: 619-28.

32. Qin Z, Dai L, Slomiany MG, Toole BP, Parsons C. Direct activation of emmprin and associated pathogenesis by an oncogenic herpesvirus. Cancer Res. 2010; 70: 3884-9.

33. Pinheiro C, Garcia EA, Morais-Santos F, Scapulatempo-Neto C, Mafra A, Steenbergen $\mathrm{RD}$, et al. Lactate transporters and vascular factors in HPV-induced squamous cell carcinoma of the uterine cervix. BMC Cancer. 2014; $14: 751$.

34. Diamond DL, Syder AJ, Jacobs JM, Sorensen CM, Walters KA, Proll SC, et al. Temporal proteome and lipidome profiles reveal hepatitis $\mathrm{C}$ virus-associated reprogramming of hepatocellular metabolism and bioenergetics. PLoS Pathog. 2010; 6: e1000719.

35. Zwerschke W, Mazurek S, Massimi P, Banks L, Eigenbrodt E, Jansen-Durr P. Modulation of type M2 pyruvate kinase activity by the human papillomavirus type 16 E7 oncoprotein. Proc Natl Acad Sci U S A. 1999; 96: 1291-6.

36. Mazurek S, Zwerschke W, Jansen-Durr P, Eigenbrodt E. Effects of the human papilloma virus HPV-16 E7 oncoprotein on glycolysis and glutaminolysis: role of pyruvate kinase type M2 and the glycolytic-enzyme complex. Biochem J. 2001; 356: 247-56.

37. Presek $\mathrm{P}$, Glossmann $\mathrm{H}$, Eigenbrodt $\mathrm{E}$, Schoner $\mathrm{W}$, Rubsamen $\mathrm{H}$, Friis RR, et al Similarities between a phosphoprotein (pp60src)-associated protein kinase of Rous sarcoma virus and a cyclic adenosine 3':5'-monophosphate-independent protein kinase that phosphorylates pyruvate kinase type M2. Cancer Res. 1980; 40: $1733-41$.

38. Presek P, Reinacher M, Eigenbrodt E. Pyruvate kinase type M2 is phosphorylated at tyrosine residues in cells transformed by Rous sarcoma virus. FEBS Lett. 1988; 242: 194-8.

39. Cooper JA, Reiss NA, Schwartz RJ, Hunter T. Three glycolytic enzymes are phosphorylated at tyrosine in cells transformed by Rous sarcoma virus. Nature. 1983; 302: 218-23.

40. Ma T, Patel H, Babapoor-Farrokhran S, Franklin R, Semenza GL, Sodhi A, et al. KSHV induces aerobic glycolysis and angiogenesis through HIF-1-dependent upregulation of pyruvate kinase 2 in Kaposi's sarcoma. Angiogenesis. 2015; 18: 477-88.

41. Wu X, Zhou Y, Zhang K, Liu Q, Guo D. Isoform-specific interaction of pyruvate kinase with hepatitis $C$ virus NS5B. FEBS Lett. 2008; 582: 2155-60.

42. Kuang Y, Schomisch SJ, Chandramouli V, Lee Z. Hexokinase and glucose-6-phosphatase activity in woodchuck model of hepatitis virus-induced hepatocellular carcinoma. Comp Biochem Physiol C Toxicol Pharmacol. 2006; 143: 225-31.

43. Ramiere C, Rodriguez J, Enache LS, Lotteau V, Andre P, Diaz O. Activity of hexokinase is increased by its interaction with hepatitis C virus protein NS5A. J Virol. 2014; 88: 3246-54.

44. Zeng $\mathrm{Q}$, Chen J, Li Y, Werle KD, Zhao RX, Quan CS, et al. LKB1 inhibits HPV-associated cancer progression by targeting cellular metabolism. Oncogene. 2017; 36:1245-55.

45. Thai M, Graham NA, Braas D, Nehil M, Komisopoulou E, Kurdistani SK, et al. Adenovirus E4ORF1-induced MYC activation promotes host cell anabolic glucose metabolism and virus replication. Cell Metab. 2014; 19: 694-701.

46. Noch E, Sariyer IK, Gordon J, Khalili K. JC virus T-antigen regulates glucose metabolic pathways in brain tumor cells. PLoS One. 2012; 7: e35054.

47. Abrantes JL, Alves CM, Costa J, Almeida FC, Sola-Penna M, Fontes CF, et al. Herpes simplex type 1 activates glycolysis through engagement of the enzyme 6-phosphofructo-1-kinase (PFK-1). Biochim Biophys Acta. 2012; 1822: 1198-206.

48. Bosca L, Mojena M, Ghysdael J, Rousseau GG, Hue L. Expression of the v-src or $\mathrm{v}$-fps oncogene increases fructose 2,6-bisphosphate in chick-embryo fibroblasts. Novel mechanism for the stimulation of glycolysis by retroviruses. Biochem J. 1986; 236: 595-9.

49. Marchand MJ, Maisin L, Hue L, Rousseau GG. Activation of 6-phosphofructo-2-kinase by pp60v-src is an indirect effect. Biochem J. 1992; 285 ( Pt 2): 413-7.

50. Jung GS, Jeon JH, Choi YK, Jang SY, Park SY, Kim SW, et al. Pyruvate dehydrogenase kinase regulates hepatitis $C$ virus replication. Sci Rep. 2016; 6: 30846

51. Cuninghame S, Jackson R, Zehbe I. Hypoxia-inducible factor 1 and its role in viral carcinogenesis. Virology. 2014; 456-457: 370-83.

52. Guo Y, Meng X, Ma J, Zheng Y, Wang Q, Wang Y, et al. Human papillomavirus 16 E6 contributes HIF-1alpha induced Warburg effect by attenuating the VHL-HIF-1alpha interaction. Int J Mol Sci. 2014; 15: 7974-86.

53. Lai D, Tan CL, Gunaratne J, Quek LS, Nei W, Thierry F, et al. Localization of HPV-18 E2 at mitochondrial membranes induces ROS release and modulates host cell metabolism. PLoS One. 2013; 8: e75625. 
54. Ripoli M, D'Aprile A, Quarato G, Sarasin-Filipowicz M, Gouttenoire J, Scrima $\mathrm{R}$, et al. Hepatitis $\mathrm{C}$ virus-linked mitochondrial dysfunction promotes hypoxia-inducible factor 1 alpha-mediated glycolytic adaptation. J Virol. 2010; 84: 647-60.

55. Darekar S, Georgiou K, Yurchenko M, Yenamandra SP, Chachami G, Simos G, et al. Epstein-Barr virus immortalization of human B-cells leads to stabilization of hypoxia-induced factor 1 alpha, congruent with the Warburg effect. PLoS One. 2012; 7: e42072.

56. Mazzon M, Peters NE, Loenarz C, Krysztofinska EM, Ember SW, Ferguson BJ, et al. A mechanism for induction of a hypoxic response by vaccinia virus. Proc Natl Acad Sci U S A. 2013; 110: 12444-9.

57. Carroll PA, Kenerson HL, Yeung RS, Lagunoff M. Latent Kaposi's sarcoma-associated herpesvirus infection of endothelial cells activates hypoxia-induced factors. J Virol. 2006; 80: 10802-12.

58. Khutornenko AA, Roudko VV, Chernyak BV, Vartapetian AB, Chumakov PM, Evstafieva AG. Pyrimidine biosynthesis links mitochondrial respiration to the p53 pathway. Proc Natl Acad Sci U S A. 2010; 107: 12828-33.

59. Schwartzenberg-Bar-Yoseph F, Armoni M, Karnieli E. The tumor suppressor p53 down-regulates glucose transporters GLUT1 and GLUT4 gene expression. Cancer Res. 2004; 64: 2627-33.

60. Doherty J, Freund R. Polyomavirus large T antigen overcomes p53 dependent growth arrest. Oncogene. 1997; 14: 1923-31.

61. Kessis TD, Slebos RJ, Nelson WG, Kastan MB, Plunkett BS, Han SM, et al. Human papillomavirus 16 E6 expression disrupts the p53-mediated cellular response to DNA damage. Proc Natl Acad Sci U S A. 1993; 90: 3988-92.

62. Parker GA, Crook T, Bain M, Sara EA, Farrell PJ, Allday MJ. Epstein-Barr virus nuclear antigen (EBNA)3C is an immortalizing oncoprotein with similar properties to adenovirus E1A and papillomavirus E7. Oncogene. 1996; 13: 2541-9.

63. Zhang R, Su J, Xue SL, Yang H, Ju LL, Ji Y, et al. HPV E6/p53 mediated down-regulation of miR-34a inhibits Warburg effect through targeting LDHA in cervical cancer. Am J Cancer Res. 2016; 6: 312-20.

64. Yogev O, Lagos D, Enver T, Boshoff C. Kaposi's sarcoma herpesvirus microRNAs induce metabolic transformation of infected cells. PLoS Pathog. 2014; 10: e1004400.

65. Lo AK, Dawson CW, Young LS, Ko CW, Hau PM, Lo KW. Activation of the FGFR1 signalling pathway by the Epstein-Barr virus-encoded LMP1 promotes aerobic glycolysis and transformation of human nasopharyngeal epithelial cells. J Pathol. 2015; 237: 238-48.

66. Jiang Y, Yan B, Lai W, Shi Y, Xiao D, Jia J, et al. Repression of Hox genes by LMP1 in nasopharyngeal carcinoma and modulation of glycolytic pathway genes by HoxC8. Oncogene. 2015; 34: 6079-91.

67. Liu B, Fang M, He Z, Cui D, Jia S, Lin X, et al. Hepatitis B virus stimulates G6PD expression through HBx-mediated Nrf2 activation. Cell Death Dis. 2015; 6: e1980.

68. Xie Q, Fan F, Wei W, Liu Y, Xu Z, Zhai L, et al. Multi-omics analyses reveal metabolic alterations regulated by hepatitis $B$ virus core protein in hepatocellular carcinoma cells. Sci Rep. 2017; 7: 41089.

69. Levy G, Habib N, Guzzardi MA, Kitsberg D, Bomze D, Ezra E, et al. Nuclear receptors control pro-viral and antiviral metabolic responses to hepatitis $\mathrm{C}$ virus infection. Nat Chem Biol. 2016; 12: 1037-45.

70. Bhatt AP, Jacobs SR, Freemerman AJ, Makowski L, Rathmell JC, Dittmer DP, et al. Dysregulation of fatty acid synthesis and glycolysis in non-Hodgkin lymphoma. Proc Natl Acad Sci U S A. 2012; 109: 11818-23.

71. Karki R, Lang SM, Means RE. The MARCH family E3 ubiquitin ligase K5 alters monocyte metabolism and proliferation through receptor tyrosine kinase modulation. PLoS Pathog. 2011; 7: e1001331. 